\title{
Interaction of the Brain-Selective Sulfotransferase SULT4A1 with Other Cytosolic Sulfotransferases: Effects on Protein Expression and Function $^{\mathbb{S}}$
}

\author{
Misgana Idris, Deanne J. Mitchell, Richard Gordon, Neelima P. Sidharthan, Neville J. Butcher, \\ and Rodney F. Minchin
}

School of Biomedical Sciences, University of Queensland, St Lucia, Queensland, Australia

Received October 20, 2019; accepted January 28, 2020

\begin{abstract}
Sulfotransferase (SULT) 4A1 is a brain-selective sulfotransferaselike protein that has recently been shown to be essential for normal neuronal development in mice. In the present study, SULT4A1 was found to colocalize with SULT1A1/3 in human brain neurons. Using immunoprecipitation, SULT4A1 was shown to interact with both SULT1A1 and SULT1A3 when expressed in human cells. Mutation of the conserved dimerization motif located in the $C$ terminus of the sulfotransferases prevented this interaction. Both ectopically expressed and endogenous SULT4A1 decreased SULT1A1/3 protein levels in neuronal cells, and this was also prevented by mutation of the dimerization motif. During differentiation of neuronal SH-SY5Y cells, there was a loss in SULT1A1/3 protein but an increase in SULT4A1 protein. This resulted in an increase in the toxicity of
\end{abstract}

\section{Introduction}

The aryl sulfotransferases (SULTs) are cytosolic enzymes that catalyze the transfer of a sulfonate group from the common cofactor $3^{\prime}$-phosphoadenosine-5' -phosphosulfate to a wide range of endogenous and exogenous substrates. In humans, there are at least 13 different SULT genes, which have been grouped into three families based on amino acid sequence homology: SULT1, SULT2, and SULT4 (Blanchard et al., 2004; Freimuth et al., 2004). The SULT1A family consists of $1 \mathrm{~A} 1,1 \mathrm{~A} 2,1 \mathrm{~A} 3$, and $1 \mathrm{~A} 4$, in which the $1 \mathrm{~A} 3$ and $1 \mathrm{~A} 4$ genes appear to have arisen from a segmental duplication that encodes the same protein (Hildebrandt et al., 2004). The SULT1A sulfotransferases are expressed widely with high abundance in the liver, lung, brain, skin, platelets, gastrointestinal tissues, and kidney (Maus et al., 1982; Campbell et al., 1987; Heroux et al., 1989; Cappiello et al., 1990; Falany et al., 1990; Nakamura et al., 1990; Hume and Coughtrie, 1994; Kudlacek et al., 1995). In humans, the SULT1A subfamily members SULT1A1 and SULT1A2 primarily conjugate xenobiotics and have a role in the activation of carcinogens (Glatt, 2000), although they also sulfonate endogenous substrates (Falany, 1997; Strott, 2002). Human SULT1A3 has high catalytic activity toward catecholamines, such as dopamine,

This work was supported by the National Health and Medical Research Council of Australia [Grant NHMRC 511175 and 1005899].

https://doi.org/10.1124/dmd.119.089714.

S This article has supplemental material available at dmd.aspetjournals.org. dopamine, a substrate for SULT1A3. Inhibition of SULT4A1 using small interference RNA abrogated the loss in SULT1A1/3 and reversed dopamine toxicity. These results show a reciprocal relationship between SULT4A1 and the other sulfotransferases, suggesting that it may act as a chaperone to control the expression of SULT1A1/3 in neuronal cells.

\section{SIGNIFICANCE STATEMENT}

The catalytically inactive sulfotransferase (SULT) 4A1 may regulate the function of other SULTs by interacting with them via a conserved dimerization motif. In neuron-like cells, SULT4A1 is able to modulate dopamine toxicity by interacting with SULT1A3, potentially decreasing the metabolism of dopamine.

ABBREVIATIONS: ERK, extracellular signal-regulated kinase; HA, hemagglutinin; MAP2, microtubule-associated protein 2; Pin1, peptidyl-prolyl cis-trans isomerase never-in-mitosis-gene-A-interacting 1; RA, retinoic acid; siRNA, small interference RNA; SULT, sulfotransferase. 
A Anterior Cingulate Cortex
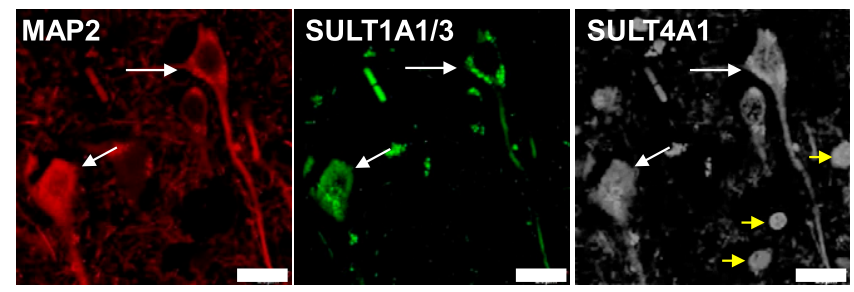

\section{B Substantia Nigra}
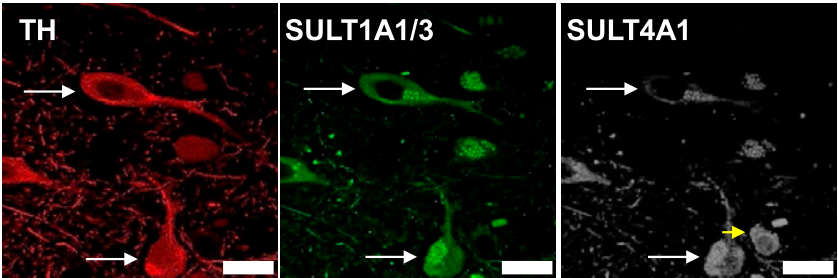

Fig. 1. SULT $1 A 1 / 3$ and SULT4A 1 proteins colocalize in human brain. (A) Anterior cingulate cortex stained with anti-MAP2 antibody (red) shows the presence of neurons. SULT1A1/3 staining is shown in the green panel, whereas SULT4A1 is shown in the gray panel. White arrows indicate colocalization of SULT1A1/3 and SULT4A1 in neuronal cells. Yellow arrows show SULT4A1 expression in cells that were negative for both SULT1A1/3 and MAP2. (B) Protein expression in the substantia nigra. Dopaminergic neurons were stained with anti-tyrosine hydroxylase (TH) antibody. White scale bar, $20 \mu \mathrm{m}$.

neuronal cells, and it has been suggested that it may be involved in regulating redox homeostasis and provide protection against mitochondrial dysfunction and oxidative stress (Hossain et al., 2019). This study further highlights the potential physiologic importance of SULT4A1 expression.

Most mammalian cytosolic sulfotransferases are found as homodimers in solution, with the mouse estrogen sulfotransferase Sult1e1 being an exception. The physiologic consequence of dimer formation is still unclear, although studies have shown differences in function and stability between the monomeric and dimeric forms (Thomae et al., 2002; Cook et al., 2010; Sidharthan et al., 2014). Crystallographic and site-directed mutagenesis experiments have shown that a conserved sequence motif of 10 residues (KxxxTVxxxE) located toward the $\mathrm{C}$ terminus of the protein is responsible for the interaction between the two sulfotransferase monomers (Petrotchenko et al., 2001). The two residues ( $\mathrm{T}$ and V) at the center of the dimerization motif make strong symmetric contacts between the monomers. Dimeric human SULT1E1 contains a dimerization motif, whereas monomeric mouse Sult 1e1 contains the sequence PE in place of TV. Mutation of TV to PE in human SULT1E1 produced a monomer, whereas mutation of mouse PE to TV created a homodimer, establishing the validity of the motif as the dimerization interface (Petrotchenko et al., 2001). Interestingly, heterodimers can also form between different SULTs containing the dimerization motif (Petrotchenko et al., 2001). Enzyme kinetic studies indicated that the two monomers do not catalyze sulfonation independently when present as a dimer (Sun and Leyh, 2010; Leyh et al., 2013). Moreover, dimerization may allow for cofactor-dependent allosteric regulation of enzyme activity (Tibbs et al., 2015).

During a yeast two-hybrid screen of a human brain library with SULT4A1, interactions between SULT4A1 and a variety of cytosolic sulfotransferases were observed, including SULT1A1 and SULT1A3, which are also found in abundance in the brain (Salman et al., 2009; James and Ambadapadi, 2013). These observations prompted the current study into the interaction of SULT4A1 with SULT1A1 and SULT1A3 using human cell models to identify protein-protein binding and determine whether any observed interactions affected the toxicity of the SULT1A3 substrate dopamine.

A

\section{Homodimerization}
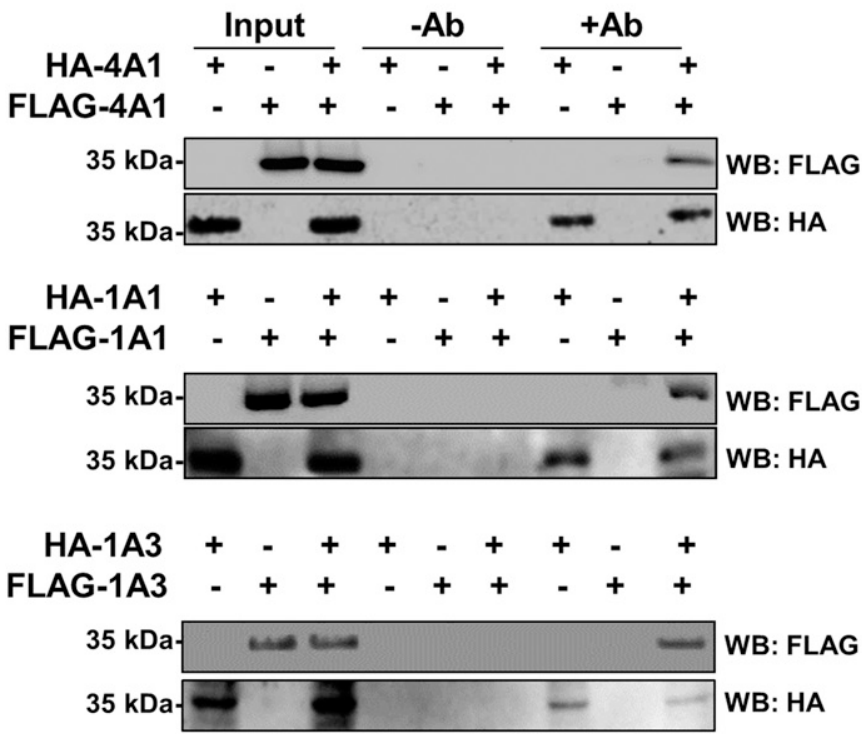

B Heterodimerization
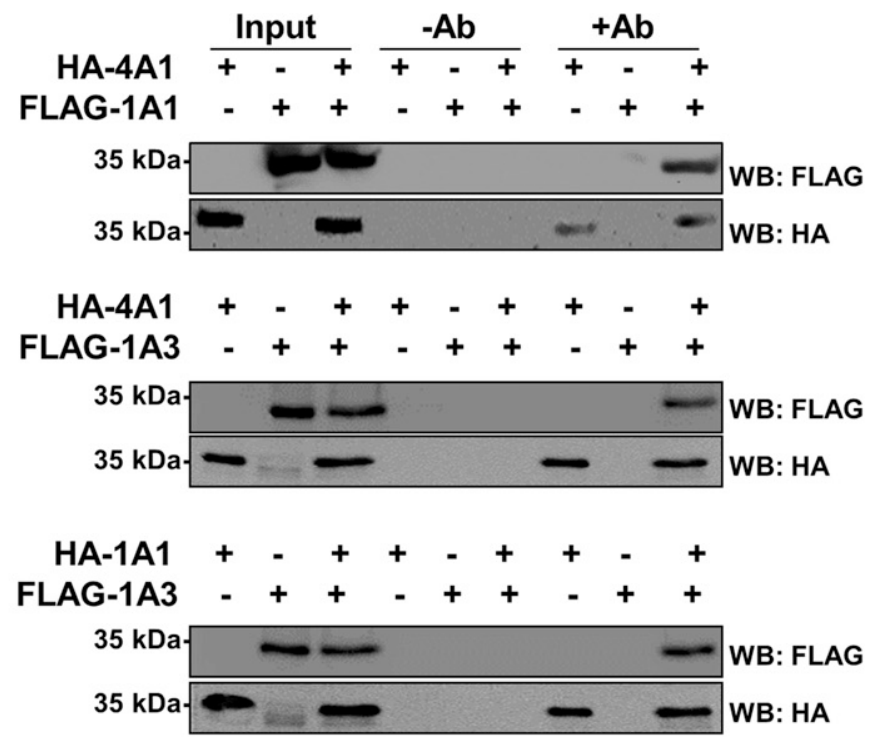

Fig. 2. SULTs form homodimers and heterodimers in HeLa cells after transient expression. (A) Cells were cotransfected with either HA- and FLAG-tagged SULT4A1 (top panels), SULT1A1 (middle panels), or SULT1A3 (bottom panels). Cells were lysed and then immunoprecipitated using anti-HA antibody and protein A-Sepharose beads. In controls, the anti-HA antibody was omitted $(-\mathrm{Ab})$. Coimmunoprecipitation of FLAG-tagged SULTs was determined by FLAG Western blot, and immunoprecipitation of HA-tagged SULTs was confirmed by HA Western blot. (B) Cells were cotransfected with HA-4A1 and FLAG-1A1 (top panels), HA4A1 and FLAG-1A3 (middle panels), or HA-1A1 and FLAG-1A3 (bottom panels) and then treated as described in (A). Results are representative of duplicate experiments. Molecular masses are shown in kilodalton. $-\mathrm{Ab}$, immunoprecipitations in the absence of anti-HA antibody; $+\mathrm{Ab}$, immunoprecipitations in the presence of anti-HA antibody; Input, protein expression in cell cytosols before immunoprecipitation (1/20); WB, Western blot. 


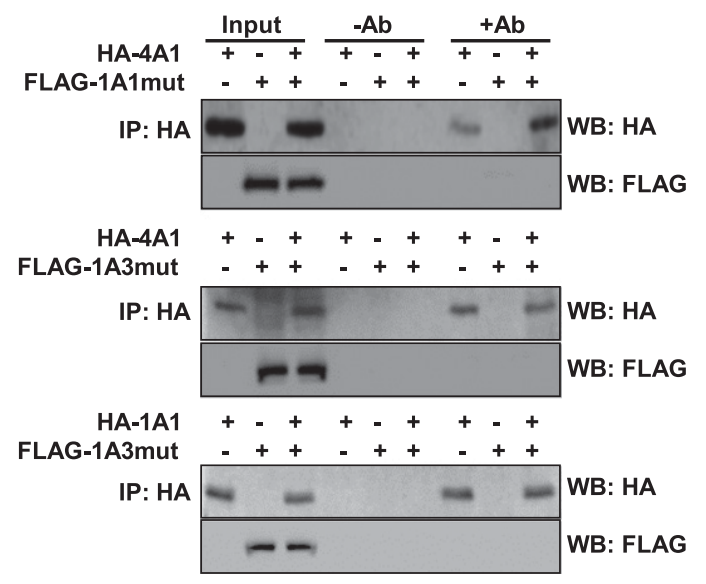

Fig. 3. Mutation of the SULT1A1/3 dimerization motif prevents heterodimer formation with SULT4A1 in HeLa cells after transient expression. Cells were cotransfected with HA-4A1 and dimerization mutant FLAG-1A1 (FLAG-1A1mut) (top panels), FLAG-1A3mut (middle panels), or with HA-1A1 and FLAG-1A3mut (bottom panels). Cells were lysed and then immunoprecipitated using anti-HA antibody and protein A-Sepharose beads. In controls, the anti-HA antibody was omitted $(-\mathrm{Ab})$. Coimmunoprecipitation of FLAG-tagged SULTs was determined by FLAG Western blot, and immunoprecipitation of HA-tagged SULTs was confirmed by HA Western blot. Results are representative of duplicate experiments. Molecular masses are shown in kilodalton. - $\mathrm{Ab}$, immunoprecipitations in the absence of anti$\mathrm{HA}$ antibody; $+\mathrm{Ab}$, immunoprecipitations in the presence of anti-HA antibody; Input, protein expression in cell cytosols before immunoprecipitation (1/20); IP, immunoprecipitation; WB, Western blot.

\section{Materials and Methods}

Cell Lines, Transient Transfections, and Cell Differentiation. HeLa, SKN-MC, and SH-SY5Y cells were obtained from the American Type Culture Collection (Manassas, VA) and were maintained in RPMI 1640 medium (Hela), Dulbecco's modified Eagle's medium: nutrient mixture F-12 (SH-SY5Y), or minimum essential medium (SK-N-MC) supplemented with 10\% FBS and incubated at $37^{\circ} \mathrm{C}$ in $5 \% \mathrm{CO}_{2}$. For transient transfections, cells were seeded in sixwell plates at $0.6 \times 10^{6}$ cells per well. Cells were transfected for 24 hours, and total transfected DNA was held constant at $4 \mu \mathrm{g}$ by addition of empty control plasmid. Plasmid DNA was transfected using LipofectAMINE 2000 reagent (Invitrogen) according to the manufacturer's instructions. SK-N-MC and SHSY5Y were differentiated into neuron-like cells in normal medium supplemented with $10 \mu \mathrm{M}$ trans-retinoic acid (RA) (Sigma-Aldrich) for 5 days and then in medium containing low serum $(1 \%$ FBS $)$ and RA $(10 \mu \mathrm{M})$ for a further 5 days.

Construct Cloning. The generation of the HA-tagged pHM6-SULT4A1 and FLAG-tagged pX3FLAG-CMV-7.1-SULT4A1 constructs have been described previously (Mitchell and Minchin, 2009). For SULT1A1 and SULT1A3, the following forward and reverse primers containing EcoRI/XbaI and HindIII/EcoRI restriction sites (underlined) were used for PCR and to clone each into pFLAGCMV-7.1 or phosphate metabolism protein 6, respectively: FP 5'-GATCGAA TTCGATGGAGCTGATCCAGGAC-3' and RP 5'-TCTAGATCTAGATCA $\overline{\text { CAGCTCAGAGCGGAAG-3' }{ }^{\prime} \text { and FP 5'-GATCAAGCTTGATGGAGCTGAT }}$ CCAGGAGACC-3' and RP 5'-GATCGAATTCTCACAGCTCAGAGCGGAA GC-3'. SULT1A1 and SULT1A3 dimerization mutants (V270E) were generated using the GeneTailor site-directed mutagenesis kit (Invitrogen) and the SULT constructs described above as templates with the following common primers: FP 5'-GACTGGAAGACCACCTTCACCGAGGCGCAGAATGAG-3' and RP 5'-CGGTGAAGGTGGTCTTCCAGTCCCCAGCCATG-3' (mutated bases underlined).

To generate p3XFLAG-CMV-10-SULT4A1 for stable transfection, SULT4A1 was excised from pFLAG-CMV-7.1 and ligated into the same sites of p3XFLAGCMV-10 (Sigma-Aldrich), which contains a G418 selection cassette. This plasmid was then used as template to create the SULT4A1 dimerization mutant $(\mathrm{KTV} \rightarrow \mathrm{EEE}$; K265E, T269E, V270E) in two steps using the GeneArt sitedirected mutagenesis kit (Invitrogen). First, the T269E and V270E mutations were introduced with primers FP 5'- GGAAGGACATCTTCGAGGAGTCCATGAA TGAGAAG-3' and RP 5'-CTTCTCATTCATGGACTCCTCGAAGATGTC CTTCC- $3^{\prime}$ (mutated bases underlined). Second, using the above construct as template, the K265E mutation was introduced with primers FP 5'-AGAGTTGGG CTGTGGGAGGACATCTTC-3' and RP 5'-GAAGATGTCCTCCCCACAG

A

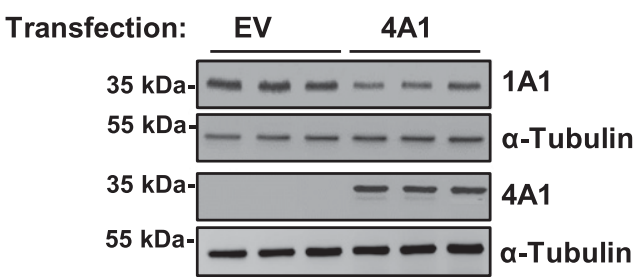

Transfection: EV 4A1

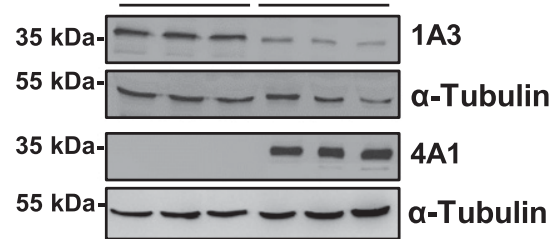

B

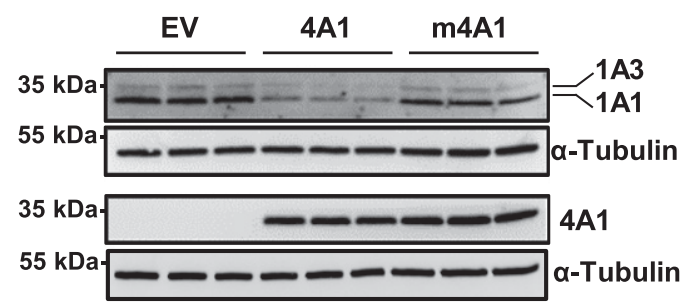

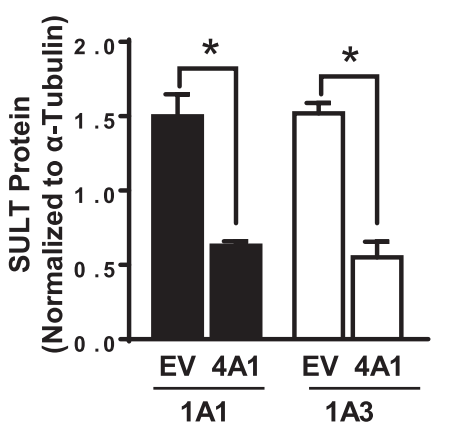

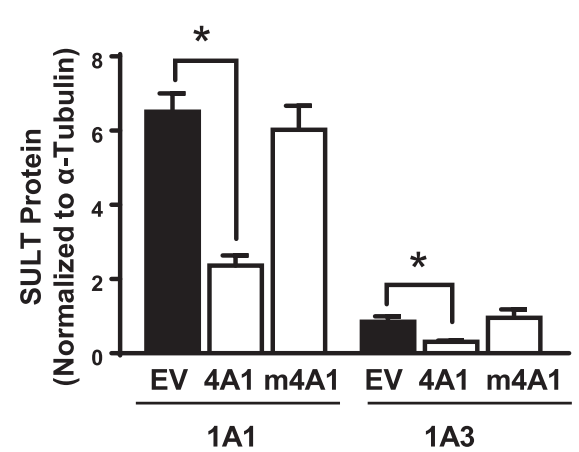

Fig. 4. SULT4A1 expression decreases levels of SULT1A1/3 proteins. (A) HeLa cells were transiently cotransfected with FLAG-SULT4A1 (4A1) or empty vector (EV) and either FLAGSULT1A1 (upper panels) or FLAG-SULT1A3 (lower panels). Cell lysates were prepared after 24 hours of transfection and Western-blotted with anti-FLAG antibody to detect expressed SULT proteins. Quantitation of Western blots was performed by densitometry using ImageJ and is shown on the graph to the right. Expression of SULT proteins was normalized to $\alpha$-tubulin. Results are mean \pm S.E.M., $n=3$. Asterisks indicate significant differences $(P<0.05)$ by one-way ANOVA. Molecular masses are shown in kilodalton. (B) Cell lysates were prepared from SK-N-MC cell lines that were stably transfected with plasmids for either wild-type FLAG-SULT4A1 (4A1), dimerization mutant FLAG-SULT4A1 (m4A1), or EV control, and levels of endogenous SULT1A1/3 proteins were determined by Western blotting with a SULT1A1/3 antibody that detects both SULT isoforms. Expression of SULT4A1 protein was verified by FLAG Western blot. Quantitation of Western blots was performed by densitometry using ImageJ and is shown on the graph. Expression of SULT proteins was normalized to $\alpha$-tubulin. Results are mean \pm S.E.M., $n=3$. Asterisks indicate significant differences $(P<0.05)$ by one-way ANOVA. Molecular masses are shown in kilodalton. 

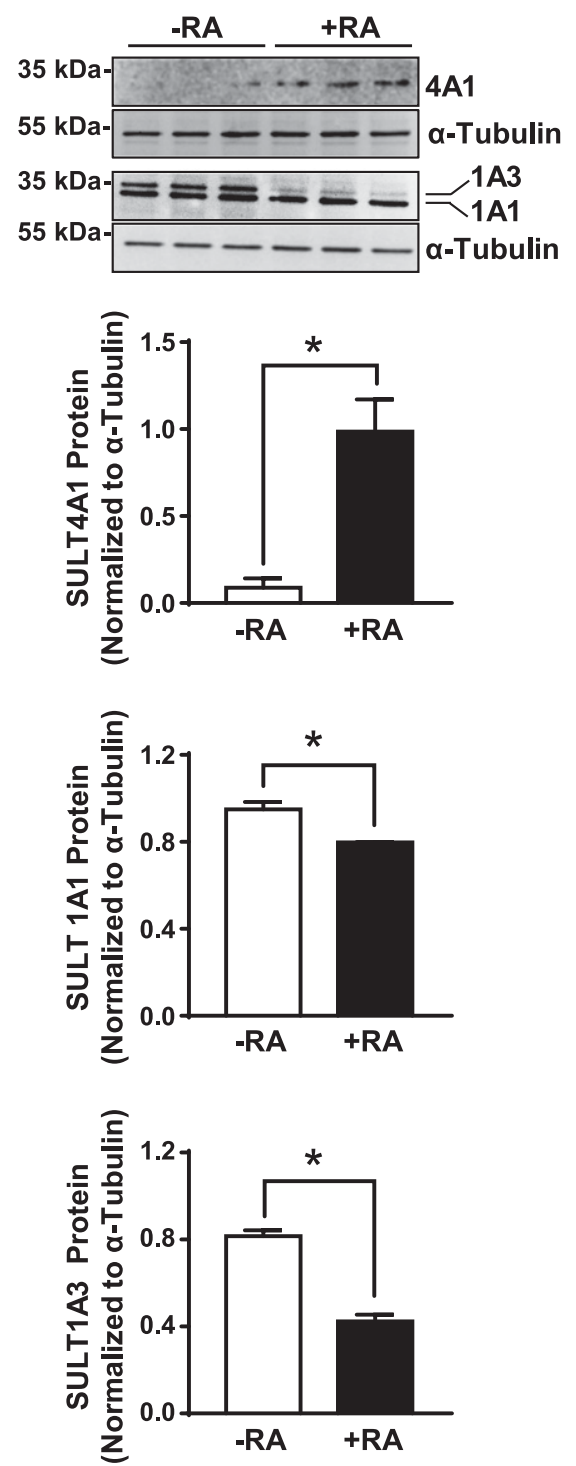

Fig. 5. Differentiation-induced upregulation of SULT4A1 expression decreases SULT1A1/3 protein levels in SK-N-MC cells. SK-N-MC cells were treated for 10 days with $10 \mu \mathrm{M}$ retinoic acid (+RA), and then endogenous SULT4A1 and SULT1A1/3 protein levels were quantified by Western blotting. Quantitation of Western blots was performed by densitometry using ImageJ and is shown on the graphs. Expression of SULT proteins was normalized to $\alpha$-tubulin. Results are mean \pm S.E.M., $n=3$. Asterisks indicate significant differences $(P<0.05)$ by Student's $t$ test compared with untreated control cells (-RA). Molecular masses are shown in kilodalton.

CCCAACTCT- $3^{\prime}$ (mutated bases underlined). The lysine at position 265 is a site of polyubiquitination and was changed to stabilize the dimerization mutant SULT4A1 protein (Sidharthan et al., 2014). All clones were verified by DNA sequencing.

Stable Cell Lines. To generate stable cell lines, SK-N-MC cells were transfected with p3XFLAG-CMV-10 plasmid containing either wild-type or dimerization mutant (KTV $\rightarrow$ EEE) SULT4A1 or empty vector using LipofectAMINE 2000 (Invitrogen). Transfected cells were then selected with $1 \mathrm{mg} / \mathrm{ml}$ Geneticin (Thermo Fisher Scientific), and single clones were isolated and expanded. Stable cell lines were maintained in medium supplemented with $0.3 \mathrm{mg} / \mathrm{ml}$ Geneticin. Expression of the SULT4A1 proteins was verified by Western blot with anti-FLAG antibody.

Immunocytochemistry. Post-mortem human brain tissue from confirmed neurologically normal individuals was obtained from the Queensland Brain Bank in Brisbane, Australia under the University of Queensland Ethics Committee approval (2012001283). Frozen cryostat sections from the anterior cingulate cortex and formalin-fixed paraffin-embedded sections $(7 \mu \mathrm{m}$-thick) from the substantia nigra were used. Antigen retrieval was performed using $0.05 \%$ citraconic anhydride $\left(\mathrm{pH} \mathrm{7.4)}\right.$ at $90^{\circ} \mathrm{C}$ for 30 minutes, and this was followed by treatment with Historeveal solution (Abcam) according to the manufacturer's instructions. Sections were then washed three times in PBS and incubated in blocking buffer (PBS containing 2\% bovine serum albumin, 5\% normal donkey serum, $0.1 \%$ Triton X-100, and $0.05 \%$ Tween-20) for 2 hours at room temperature. Sections were then washed once in PBS and incubated with primary antibodies for SULT4A1 (Proteintech, diluted 1:1000) and SULT1A1/1A3 (gift from Professor Mike Coughtrie, The University of British Columbia, Vancouver, Canada; diluted 1:1000) overnight at $4^{\circ} \mathrm{C}$ in antibody buffer (PBS with $2 \%$ bovine serum albumin and $2 \%$ normal donkey serum). Tyrosine hydroxylase (diluted 1 : 2000; Cell Signaling Technology) was used as a dopaminergic neuron marker and
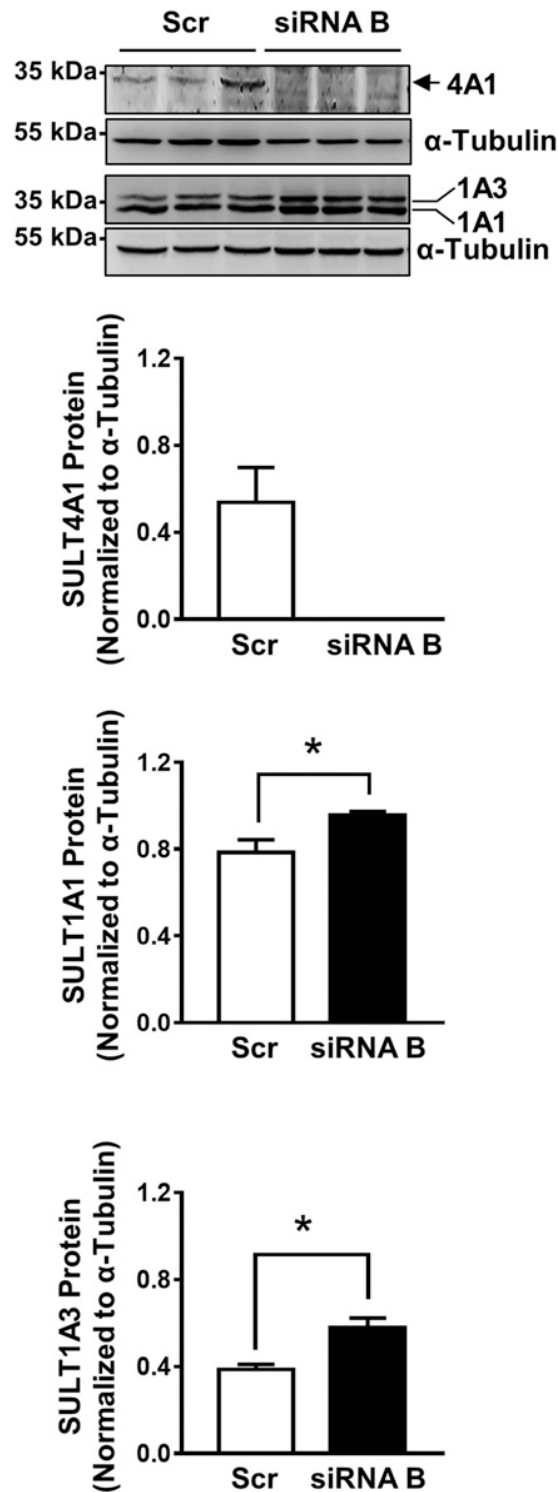

Fig. 6. Knockdown of SULT4A1 with siRNA in differentiated SK-N-MC cells results in increased SULT1A1/3 protein levels. Cells were treated for 7 days with 10 $\mu \mathrm{M}$ retinoic acid and then transfected with $20 \mathrm{nM}$ of either scrambled (Scr) or SULT4A1 siRNA (siRNA B or C) in the presence of retinoic acid for an additional 3 days. Endogenous SULT4A1 and SULT1A1/3 protein levels were determined by Western blotting. Quantitation of Western blots was performed by densitometry using ImageJ and is shown on the graphs below. Expression of SULT proteins was normalized to $\alpha$-tubulin. Results are mean \pm S.E.M., $n=3$. Asterisks indicate significant difference $(P<0.05)$ by Student's $t$ test from cells treated with scrambled siRNA. Molecular masses are shown in kilodalton. 
A

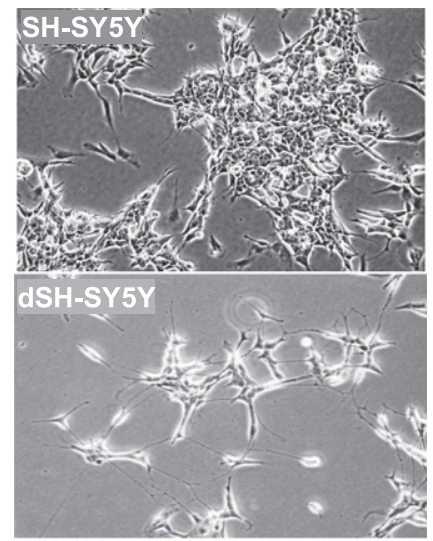

B

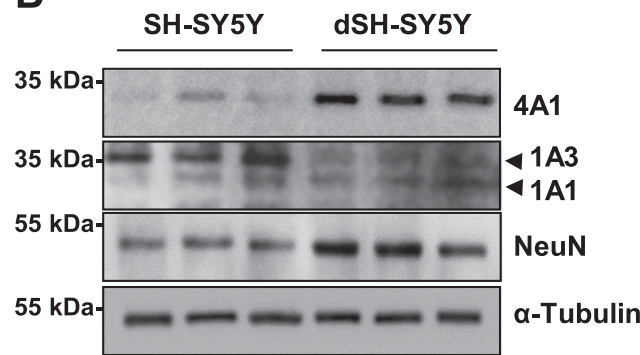

Fig. 7. SULT expression in differentiated SH-SY5Y cells. (A) Light microscopy showing SH-SY5Y cells before (SH-SY5Y) and after differentiation (dSH-SY5Y) with $10 \mu \mathrm{M}$ retinoic acid for 10 days. (B) Cell lysates of undifferentiated ( $\mathrm{SH}-$ SY5Y) and differentiated (dSH-SY5Y) cells were Western-blotted for endogenous SULT4A1 and SULT1A1/3 proteins. Neuronal nuclei (NeuN) was blotted as a marker of neuronal differentiation, and $\alpha$-tubulin was a loading control. Molecular masses are shown in kilodalton.

microtubule-associated protein 2 (MAP2) (diluted 1:2000; Cell Signaling Technology) was used as a pan-neuronal marker for colocalization studies. After five washes in PBS, sections were incubated with the respective Alexa dye-conjugated secondary antibodies (diluted 1:5000) for 2 hours at room temperature. Sections were then washed 5 times in PBS and treated using the Autofluorescence Eliminator Reagent (2160; Merck Millipore) for 5 minutes to eliminate lipofuscin-derived autofluorescence. Sections were then mounted using Prolong Gold antifade reagent (Molecular Probes) for confocal microscopy.

Coimmunoprecipitation Assays. HeLa cells were transfected as described above with various combinations of plasmids that expressed FLAG-tagged and HA-tagged SULT proteins. Transiently transfected cells $\left(1 \times 10^{6}\right)$ were washed in PBS, and whole-cell extracts were prepared by incubation in $500 \mu 1$ NP40 lysis buffer (1\% NP40; $50 \mathrm{mM}$ Tris, pH 8.0; $150 \mathrm{mM} \mathrm{NaCl} ; 5 \mathrm{mM} \mathrm{NaF} ; 1 \mathrm{mM}$ $\mathrm{Na}_{3} \mathrm{VO}_{4} ; 10 \mathrm{mM} \beta$-glycerophosphate; $1 \mathrm{mM}$ EDTA) and protease inhibitor cocktail (Sigma-Aldrich) for 15 minutes on ice. Lysates were cleared by centrifugation at $14,000 \mathrm{~g}$ for 10 minutes at $4^{\circ} \mathrm{C}$, and samples were incubated with or without rabbit anti-HA antibody (diluted 1:200; Sigma-Aldrich) for 2 hours at $4^{\circ} \mathrm{C}$, after which $30 \mu$ protein A-Sepharose beads (Sigma-Aldrich) were added, and the samples were incubated for a further 1 hour at $4^{\circ} \mathrm{C}$. Beads were washed $3 \times$ with wash buffer [0.5\% NP40, $50 \mathrm{mM}$ Tris $(\mathrm{pH} 8.0), 150 \mathrm{mM}$ $\mathrm{NaCl}]$ and then boiled for 5 minutes in Laemmli buffer. Samples $(20 \mu l)$ were separated by SDS-PAGE, transferred to nitrocellulose membranes, and immunoblotted with mouse anti-FLAG M2 horseradish peroxidase-conjugated antibody (diluted 1:5000; Sigma-Aldrich) to detect FLAG-SULT binding. To validate HASULT pulldown, blots were incubated with mouse anti-HA antibody (diluted 1: 1000; Sigma-Aldrich) followed by anti-mouse horseradish peroxidase-conjugated secondary IgG (diluted 1:30,000; Jackson ImmunoResearch Laboratories). Protein bands were visualized with Immu-Star ECL reagent (Bio-Rad Laboratories) as per the manufacturer's instructions.

Western Blots. At the times indicated, cells were washed twice with cold PBS, lysed directly in Laemmli buffer, and boiled for 5 minutes. Samples (10-20 $\mu \mathrm{g}$ protein) were separated by SDS-PAGE, transferred to nitrocellulose membranes, and immunoblotted for FLAG- and HA-tagged SULT proteins, endogenous SULT1A1/3 (anti-SULT1A1/3 antibody detects both isoforms and was a gift from Professor Mike Coughtrie, The University of British Columbia, Vancouver, Canada; diluted 1:1000), SULT4A1 (Proteintech, diluted 1:1000), and $\alpha$-tubulin (diluted 1:2000; Cell Signaling Technology) as previously described (Sidharthan et al., 2013, 2014).

Small Interference RNA Studies. SH-SY5Y cells were transiently transfected with small interference RNAs (siRNAs) directed against SULT4A1 (SR308535 A, B, and C; Origene Technologies) for a total of 72 hours using LipofectAMINE RNAiMAX (Invitrogen) according to manufacturer's protocol. A universal scrambled siRNA (SR30004; Origene Technologies) was used as a negative control. All siRNAs were used at a final concentration of $20 \mathrm{nM}$. SULT4A1 gene knockdown was evaluated by Western blot.

Dopamine Toxicity. SH-SY5Y cells were seeded in 96-well plates at a density of $2000 \mathrm{cells} / \mathrm{well}$ and allowed to adhere overnight. The cells were then treated with up to $1 \mathrm{mM}$ dopamine (Sigma-Aldrich) in culture medium for 48 hours. Cell viability was then assessed using the CyQuant Cell Proliferation Assay kit (Invitrogen), according to the manufacturer's protocol.

Statistical Analysis. Data are presented as mean \pm S.E.M. unless otherwise noted. Significant differences were determined by Student's $t$ test $(P<0.05)$ or ANOVA as indicated. $\mathrm{IC}_{50}$ values were estimated by fitting the dopamine toxicity data to a dose-response curve using nonlinear least-squares regression (GraphPad Prism).

\section{Results}

Colocalization of SULT4A1 and SULT1A1/3 in Human Brain. The physiologic relevance of any interaction between the sulfotransferases is predicated on whether the proteins are expressed in the same cell type(s). Therefore, immunocytochemistry was performed with human brain tissue from two different regions to determine whether SULT4A1 colocalizes with SULT1A1/3 (Fig. 1). The SULT1A1/3 antibody recognizes both proteins (Riches et al., 2009), so it was not possible to determine whether the SULT1A1/3 staining represents either or both sulfotransferases. Nevertheless, in the anterior cingulate cortex, all cells that expressed SULT1A1/3 also expressed SULT4A1 (Fig. 1A). Moreover, these cells were of neuronal origin, as identified by costaining with anti-MAP2 antibody. There were some cells that expressed SULT4A1 but were negative for SULT1A1/3 (Fig. 1A, yellow arrows). These cells were also negative for MAP2 staining, suggesting they may be of non-neuronal origin. A similar colocalization was seen in the substantia nigra, where both of the sulfotransferases were expressed in dopaminergic neurons, which were identified by costaining with antityrosine hydroxylase antibody (Fig. 1B). These results demonstrate colocalization of SULT4A1 with SULT1A1 and/or SULT1A3 in human brain and suggest that any interaction between these proteins may produce a physiologic effect.

SULT4A1, SULT1A1, and SULT1A3 Proteins Form Homodimers and Heterodimers that Require the Sulfotransferase Dimerization Motif. Using HeLa cells that do not express SULT1A1/3 or SULT4A1, FLAG-tagged and HA-tagged SULTs were coexpressed and then coimmunoprecipitated with anti-HA antibody. Initially, each SULT was coexpressed with itself to determine homodimerization (Fig. 2A). Western blots for FLAG-tagged SULTs that coimmunoprecipitated with HA-tagged SULTs showed that all three SULTs formed homodimers in HeLa cells. Western blots of samples prior to immunoprecipitation (Input) confirmed expression of each FLAG-tagged SULT in the cells (Fig. 2A, lanes 2 to 3 in each blot). Furthermore, both expression (Input) and immunoprecipitation of the HA-tagged proteins were also confirmed (Fig. 2A, lower panels). Omitting the HA antibody showed there was no nonspecific binding to protein A beads (Fig. 2A, lanes 4-6 in each blot).

Coexpression of the different SULTs as FLAG-tagged and HAtagged proteins was then performed to detect heterodimerization. All 

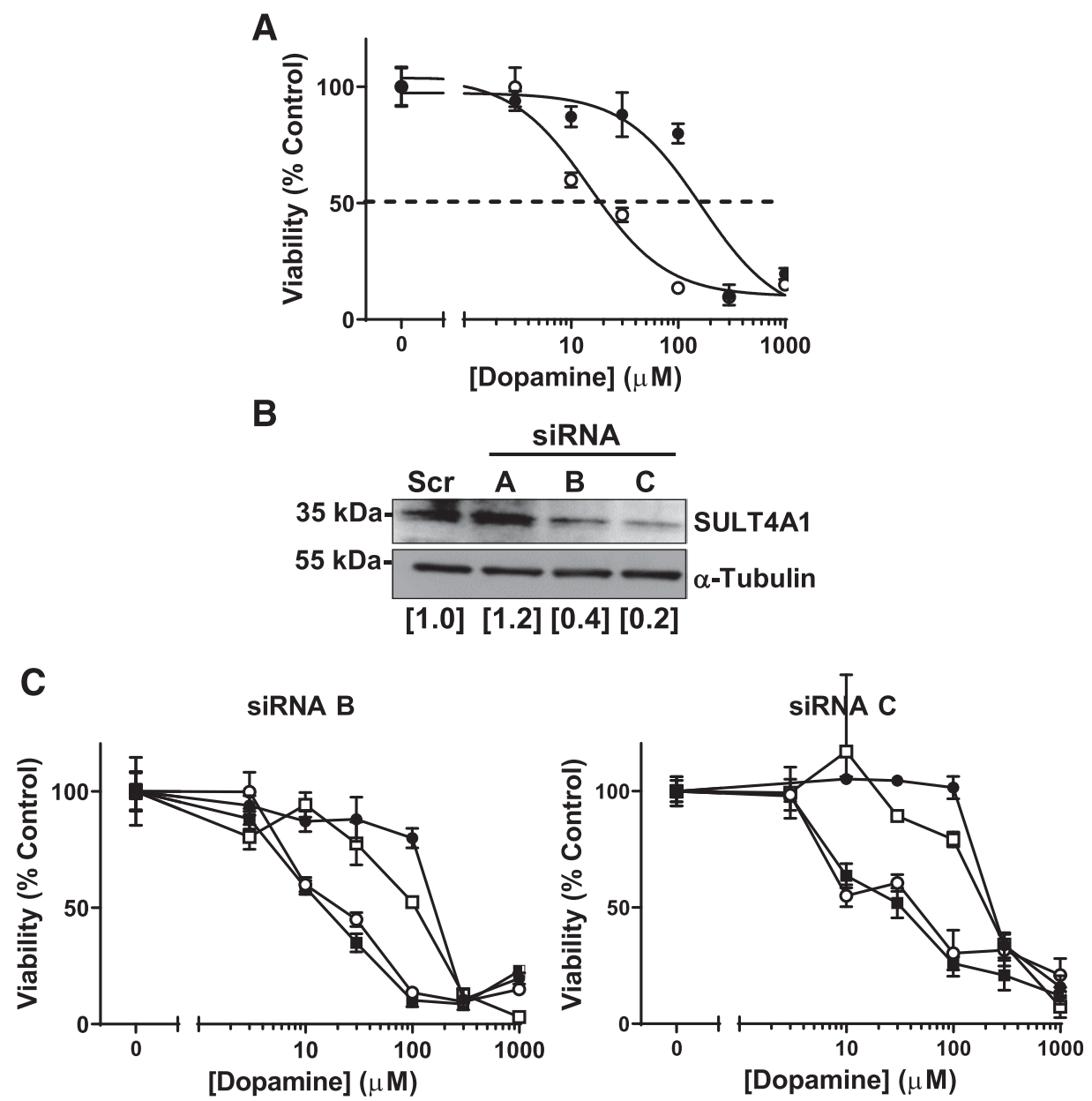

SH-SY5Y

O dSH-SY5Y

dSH-SY5Y + Scr

dSH-SY5Y + SIRNA
Fig. 8. Knockdown of SULT4A1 with siRNA reduces dopamine toxicity in differentiated SHSY5Y cells. (A) Toxicity of dopamine in SH-SY5Y cells before $(\mathbf{)}$ and after (dSH-SY5Y, $O$ ) differentiation with RA for 10 days. (B) SH-SY5Y cells were differentiated for 7 days and then transfected with $20 \mathrm{nM}$ of either scrambled (Scr) or SULT4A1 siRNA (siRNA A, B, or C) in the presence of retinoic acid for an additional 3 days. Cells were lysed after 72 hours of siRNA transfection and subjected to Western blot to verify knockdown of SULT4A1. Quantification of knockdown is shown in parentheses below the blots and is expressed relative to Scr and normalized to $\alpha$-tubulin. Only siRNA B and C showed knockdown. (C) Undifferentiated (-), differentiated (C), differentiated treated with scrambled siRNA (-), and differentiated treated with SULT4A1 siRNAs B (left graph) or C (right graph) (๑) SH-SY5Y cells were seeded into 96-well plates at 2000 cells/well and allowed to adhere overnight. The cells were then treated with up to $1 \mathrm{mM}$ dopamine, and viability was assessed using a CyQuant proliferation assay kit. Results are mean \pm S.E.M., $n=4$. three proteins interacted with each other as detected by immunoprecipitation (Fig. 2B, last lanes). To determine whether the dimerization motif was required for protein-protein interactions, both SULT1A1 and SULT1A3 were mutated to the sequence found in mouse Sult1e1, which does not form homodimers (T269P, V270E) (Petrotchenko et al., 2001). Mutants bound neither to SULT4A1 nor to each other (Fig. 3, last lanes). Taken together, these results suggest that SULT4A1, SULT1A1, and SULT1A3 can interact with each other in the context of the whole cell. Moreover, this interaction appears to occur via the conserved dimerization motif in the $\mathrm{C}$ terminus of each protein.

A yeast-2-hybrid assay and an in vitro pulldown of glutathione $S$-transferase-tagged recombinant proteins were also investigated to confirm the immunoassay results. In both assays, SULT4A1 was shown to bind to SULT1A1/3 (Supplemental Fig. 1; Supplemental Table 1, respectively).

SULT4A1 Protein Expression Decreases SULT1A1/3 Protein Levels. HeLa cells were transiently transfected with SULT4A1 and either SULT1A1 or SULT1A3, after which protein levels were determined by Western blotting. In the presence of SULT4A1, expression of SULT1A1 and SULT1A3 was significantly attenuated by $60 \%$ and $64 \%$, respectively, compared with empty vector controls (Fig. 4A). To determine whether a similar effect is seen with endogenous SULTs, SK$\mathrm{N}-\mathrm{MC}$ cells were used because these cells constitutively express both SULT1A1 and SULT1A3 but very low levels of SULT4A1 (Fig. 4B). In cells stably transfected with SULT4A1, endogenous SULT1A1 and SULT1A3 proteins were decreased by $90 \%$ and $85 \%$, respectively
(Fig. 4B). However, when cells were stably transfected with a SULT4A1 mutant, there was no change in SULT1A1 or SULT1A3 protein (Fig. 4C). These experiments show that the presence of SULT4A1 leads to a decrease in SULT1A1/3 protein, most likely after proteinprotein interactions.

It has been shown previously that SULT4A1 is induced after differentiation of SK-N-MC cells with retinoic acid (Sidharthan et al., 2013). Since SULT1A1 and SULT1A3 are constitutively expressed in these cells, this was a good model to determine whether upregulation of endogenous SULT4A1 affects endogenous SULT1A1/3 expression. The levels of SULT1A1/3 protein were quantified before and after treatment with $10 \mu \mathrm{M}$ retinoic acid for 10 days (Fig. 5). There was an 11fold increase in SULT4A1 protein after differentiation, which was accompanied by $20 \%$ and $50 \%$ decreases in SULT1A1 and SULT1A3 proteins, respectively $(P<0.05$, Student's $t$ test). To determine whether this change was due to the increase in SULT4A1, cells were differentiated with retinoic acid and then treated with siRNA directed against SULT4A1 mRNA. Figure 6 (upper blot) shows efficient downregulation of SULT4A1 by the siRNA compared with a scrambled sequence. After treatment, the loss in SULT1A1/3 seen in Fig. 5 was reversed. These results show that endogenous SULT4A1 may regulate the level of SULT1A1/3 protein expression in cells.

SULT4A1 Upregulation During Neuronal Differentiation Enhances Dopamine Toxicity. Dopamine is toxic to most cells, but this can be attenuated by SULT1A3, which metabolizes dopamine to dopamine sulfate (Sidharthan et al., 2013). Thus, a change in SULT1A3 
expression leads to a change in cell survival. Unlike SK-N-MC cells, SH-SY5Y cells express low levels of SULT4A1 and SULT1A1 but high levels of SULT1A3. After $10 \mu \mathrm{M}$ retinoic acid treatment of 10 days, the SH-SY5Y cells showed characteristic dendrite formation consistent with a neuronal-like phenotype (Fig. 7A). Moreover, the neuron-specific marker neuronal nuclei was up-regulated (Fig. 7B). Similar to that seen in the SK-N-MC cells, SULT4A1 protein increased, whereas SULT1A3 protein decreased upon differentiation. Expression of SULT1A1 was low both before and after treatment (Fig. 7B).

To determine whether the downregulation of SULT1A3 by endogenous SULT4A1 affects dopamine toxicity, SH-SY5Y cells were treated with increasing concentrations of dopamine before and after differentiation. The toxicity of dopamine appeared to be inversely related to SULT1A3 expression levels. This is seen in Fig. 8A, in which the $\mathrm{IC}_{50}$ of dopamine decreased from $137 \pm 16 \mu \mathrm{M}$ in undifferentiated cells (high SULT1A3) to $15 \pm 1 \mu \mathrm{M}$ after retinoic acid treatment (low SULT1A3). To determine whether this increase in toxicity was related to an increase in SULT4A1, the differentiated cells were treated with siRNA for SULT4A1 or a scrambled sequence as a control. Two independent siRNAs were used (B and C), both of which decreased SULT4A1 protein over 72 hours (Fig. 8B). Moreover, both siRNAs reversed the increase in dopamine toxicity to that seen in the undifferentiated cells (Fig. 8C). By contrast, the scrambled sequence had no effect on toxicity.

\section{Discussion}

Despite the discovery of SULT4A1 in the brain almost 20 years ago, there has been little work to uncover its role in mammalian cells. When its expression was inhibited in a zebrafish model, distinct phenotypic changes were seen, including sedentary behavior (Crittenden et al., 2015), and upregulation of genes associated with phototransduction (Crittenden et al., 2014). In a mouse gene knockout model, a severe phenotype was observed with animals surviving only 20-25 days postbirth (Garcia et al., 2018). A recent study showed that SULT4A1 expression in SH-SY5Y cells altered mitochondrial function and protected the cells from hydrogen peroxide-induced cell death (Hossain et al., 2019). Interestingly, it also reported that SULT4A1 was colocalized with the mitochondrial outer membrane translocase protein Translocase of outer membrane $20 \mathrm{kDa}$ subunit, which forms part of a complex that imports proteins from the cytosol into mitochondria. The possible interaction of SULT4A1 with the translocase of outer membrane complex may be important for its role in altering mitochondrial function. In the present study, SULT4A1 colocalized with SULT1A1/3 in human neurons in at least two separate regions of the brain. SULT4A1 was also present in non-neuronal cells in the absence of the other sulfotransferases. SULT4A1 heterodimerized with both SULT1A1 and SULT1A3, and this interaction required an intact dimerization motif. This is the first study to identify heterodimerization between these proteins. Using ectopic expression of the sulfotransferases as well as changes in endogenous levels during cell differentiation, SULT4A1 was shown to decrease both SULT1A1 and SULT1A3 protein levels. For SULT1A3, this resulted in an increase in the toxicity of dopamine, a substrate for the enzyme. Collectively, these results show a reciprocal relationship between SULT4A1 and SULT1A1/3 in neuronal cells. The mechanism behind SULT4A1-induced SULT1A1/ 3 protein loss remains unclear and requires further study. One possible explanation may be that heterodimerization of the SULTs increases protein degradation, which is supported by the fact that mutation of the dimerization site prevents protein loss.

There is an emerging picture of SULT4A1 regulation as a complex cellular process involving mRNA splicing, post-translational modification,

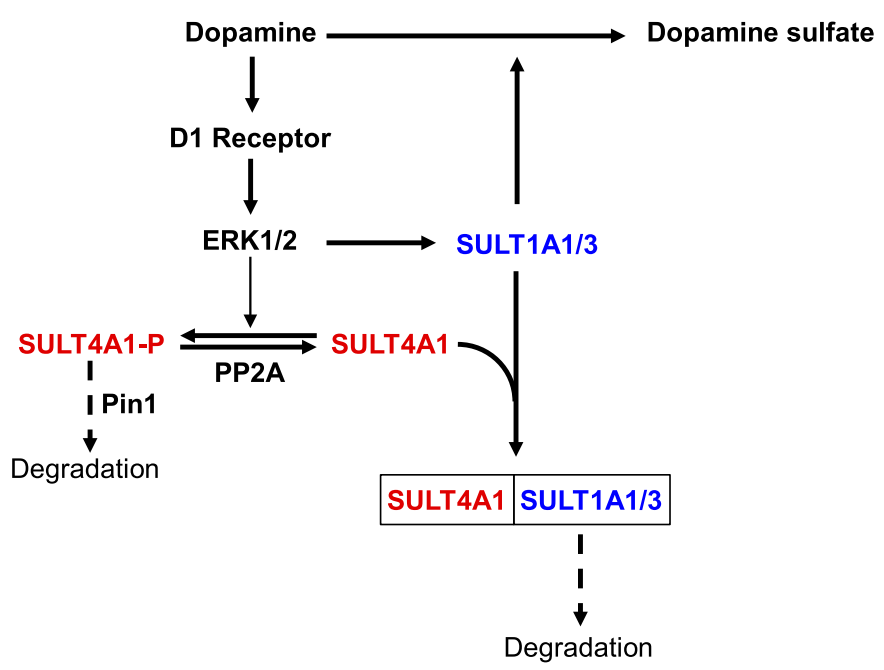

Fig. 9. Pathways for the regulation of SULT4A1 and SULT1A1/3. ERK1/2 phosphorylates SULT4A1 to generate a Pin1-binding site, which leads to protein degradation. The phosphorylated SULT4A1 is dephosphorylated by the protein phosphatase 2A (PP2A). ERK1/2 is also involved in the transcriptional regulation of SULT1A1/3 in a dopamine-dependent manner. Dopamine activates the D1 receptor to upregulate ERK1/2 activity. The increased SULT1A3 then feeds back onto dopamine through its metabolism to dopamine sulfate. Finally, SULT4A1 can dimerize with SULT1A1/3 to promote its degradation and loss of activity.

and protein-protein interactions. SULT4A1 mRNA is found in most tissues in the body but primarily as an aberrant transcript that does not translate a stable protein (Sidharthan et al., 2014). After correct splicing that removes a pseudoexon located between exons 6 and 7, the fulllength stable protein is generated. Thus, SULT4A1 protein levels are regulated at a post-transcriptional level through alternative splicing. SULT4A1 expression is also regulated by both transcriptional and posttranslational processes (Mitchell and Minchin, 2009; Butcher et al., 2010). In neuronal-like cells, SULT4A1 transcription is under the control of the cAMP response element-binding protein and the activating transcription factor-2 (Butcher et al., 2010). Posttranslationally, the protein is phosphorylated in an ERK1/2-dependent manner, which promotes binding of the peptidyl-prolyl cis-trans isomerase Pin1 and subsequent SULT4A1 degradation. These pathways are summarized in Fig. 9.

In a previous study using SK-N-MC cells, low dopamine concentrations were shown to increase SULT1A1 and SULT1A3 transcription through activation of the D1 receptor (Sidharthan et al., 2013). Part of this pathway involved upregulation of mitogen-activated protein kinase kinase-ERK1/2 signaling. Thus, ERK1/2 is a point of convergence for the regulation of both SULT1A1/3 and SULT4A1 (Fig. 9). However, although ERK1/2 upregulates SULT1A1/3, it downregulates SULT4A1. The dopamine-ERK1/2-SULT1A3 axis may provide a feedback loop to regulate dopamine levels in the brain. Controlling SULT4A1 protein levels may be an additional pathway to ensure adequate SULT1A3 protein in the presence of excess dopamine. This may be an important observation that warrants further investigation. In addition, there are a number of drugs that can modulate ERK1/2 activity, including serotonin (Li et al., 2010), amphetamines (Choe and Wang, 2002), isoproterenol (Du et al., 2010), and cocaine (Hoffmann et al., 2012). Whether these treatments alter the levels of sulfotransferases in the brain remains to be determined.

\section{Acknowledgments}

The authors thank Professor Mike Coughtrie, The University of British Columbia, for the generous gift of SULT1A1/3 antibody. 


\section{Authorship Contributions}

Participated in research design: Butcher, Minchin.

Conducted experiments: Idris, Mitchell, Gordon, Sidharthan, Butcher.

Performed data analysis: Idris, Minchin.

Wrote or contributed to the writing of the manuscript: Idris, Butcher,

Minchin.

Conflict of interest. The authors declare that there are no competing interests associated with the manuscript.

\section{References}

Allali-Hassani A, Pan PW, Dombrovski L, Najmanovich R, Tempel W, Dong A, Loppnau P, Martin F, Thornton J, Edwards AM, et al. (2007) Structural and chemical profiling of the human cytosolic sulfotransferases [published correction appears in PLoS Biol (2007) 5:e165]. PLoS Biol 5:e97.

Blanchard RL, Freimuth RR, Buck J, Weinshilboum RM, and Coughtrie MW (2004) A proposed nomenclature system for the cytosolic sulfotransferase (SULT) superfamily. Pharmacogenetics 14:199-211.

Brennan MD and Condra J (2005) Transmission disequilibrium suggests a role for the sulfotransferase-4A1 gene in schizophrenia. Am J Med Genet B Neuropsychiatr Genet 139B:69-72.

Brix LA, Barnett AC, Duggleby RG, Leggett B, and McManus ME (1999) Analysis of the substrate specificity of human sulfotransferases SULT1A1 and SULT1A3: site-directed mutagenesis and kinetic studies. Biochemistry 38:10474-10479.

Butcher NJ, Mitchell DJ, Burow R, and Minchin RF (2010) Regulation of mouse brain-selective sulfotransferase sult4a1 by cAMP response element-binding protein and activating transcription factor-2. Mol Pharmacol 78:503-510.

Campbell NR, Van Loon JA, Sundaram RS, Ames MM, Hansch C, and Weinshilboum R (1987) Human and rat liver phenol sulfotransferase: structure-activity relationships for phenolic substrates. Mol Pharmacol 32:813-819.

Cappiello M, Giuliani L, and Pacifici GM (1990) Differential distribution of phenol and catechol sulphotransferases in human liver and intestinal mucosa. Pharmacology 40:69-76.

Choe ES and Wang JQ (2002) CaMKII regulates amphetamine-induced ERK1/2 phosphorylation in striatal neurons. Neuroreport 13:1013-1016.

Condra JA, Neibergs H, Wei W, and Brennan MD (2007) Evidence for two schizophrenia susceptibility genes on chromosome 22q13. Psychiatr Genet 17:292-298.

Cook IT, Leyh TS, Kadlubar SA, and Falany CN (2010) Lack of substrate inhibition in a monomeric form of human cytosolic SULT2A1. Horm Mol Biol Clin Investig 3:357-366.

Crittenden F, Thomas H, Ethen CM, Wu ZL, Chen D, Kraft TW, Parant JM, and Falany CN (2014) Inhibition of SULT4A1 expression induces up-regulation of phototransduction gene expression in 72-hour postfertilization zebrafish larvae. Drug Metab Dispos 42:947-953.

Crittenden F, Thomas HR, Parant JM, and Falany CN (2015) Activity suppression behavior phenotype in SULT4A1 frameshift mutant zebrafish. Drug Metab Dispos 43:1037-1044.

Du T, Li B, Li H, Li M, Hertz L, and Peng L (2010) Signaling pathways of isoproterenol-induced ERK1/2 phosphorylation in primary cultures of astrocytes are concentration-dependent. J Neurochem 115:1007-1023.

Falany CN (1997) Enzymology of human cytosolic sulfotransferases. FASEB J 11:206-216.

Falany CN, Vazquez ME, Heroux JA, and Roth JA (1990) Purification and characterization of human liver phenol-sulfating phenol sulfotransferase. Arch Biochem Biophys 278:312-318.

Falany CN, Xie X, Wang J, Ferrer J, and Falany JL (2000) Molecular cloning and expression of novel sulphotransferase-like cDNAs from human and rat brain. Biochem J 346:857-864.

Freimuth RR, Wiepert M, Chute CG, Wieben ED, and Weinshilboum RM (2004) Human cytosolic sulfotransferase database mining: identification of seven novel genes and pseudogenes. Pharmacogenomics $J$ 4:54-65.

Garcia PL, Hossain MI, Andrabi SA, and Falany CN (2018) Generation and characterization of SULT4A1 mutant mouse models. Drug Metab Dispos 46:41-45.

Glatt H (2000) Sulfotransferases in the bioactivation of xenobiotics. Chem Biol Interact 129: 141-170.

Heroux JA, Falany CN, and Roth JA (1989) Immunological characterization of human phenol sulfotransferase. Mol Pharmacol 36:29-33.

Hildebrandt MA, Carrington DP, Thomae BA, Eckloff BW, Schaid DJ, Yee VC, Weinshilboum RM, and Wieben ED (2007) Genetic diversity and function in the human cytosolic sulfotransferases. Pharmacogenomics $J$ 7:133-143.

Hildebrandt MA, Salavaggione OE, Martin YN, Flynn HC, Jalal S, Wieben ED, and Weinshilboum RM (2004) Human SULT1A3 pharmacogenetics: gene duplication and functional genomic studies. Biochem Biophys Res Commun 321:870-878.
Hoffmann HM, Nadal R, Vignes M, and Ortiz J (2012) Chronic cocaine self-administration modulates ERK1/2 and CREB responses to dopamine receptor agonists in striatal slices. Addict Biol 17:565-575.

Hossain MI, Marcus JM, Lee JH, Garcia PL, Gagné JP, Poirier GG, Falany CN, and Andrabi SA (2019) SULT4A1 protects against oxidative-stress induced mitochondrial dysfunction in neuronal cells. Drug Metab Dispos 47:949-953.

Hume R and Coughtrie MW (1994) Phenolsulphotransferase: localization in kidney during human embryonic and fetal development. Histochem $J$ 26:850-855.

James MO and Ambadapadi S (2013) Interactions of cytosolic sulfotransferases with xenobiotics. Drug Metab Rev 45:401-414.

Kudlacek PE, Anderson RJ, Liebentritt DK, Johnson GA, and Huerter CJ (1995) Human skin and platelet minoxidil sulfotransferase activities: biochemical properties, correlations and contribution of thermolabile phenol sulfotransferase. J Pharmacol Exp Ther 273:582-590.

Lewis AG and Minchin RF (2009) Lack of exonic sulfotransferase 4A1 mutations in controls and schizophrenia cases. Psychiatr Genet 19:53-55.

Leyh TS, Cook I, and Wang T (2013) Structure, dynamics and selectivity in the sulfotransferase family. Drug Metab Rev 45:423-430.

Li B, Zhang S, Li M, Hertz L, and Peng L (2010) Serotonin increases ERK1/2 phosphorylation in astrocytes by stimulation of 5-HT2B and 5-HT2C receptors. Neurochem Int 57:432-439.

Maus TP, Pearson RK, Anderson RJ, Woodson LC, Reiter C, and Weinshilboum RM (1982) Rat phenol sulfotransferase. Assay procedure, developmental changes, and glucocorticoid regulation. Biochem Pharmacol 31:849-856.

Minchin RF, Lewis A, Mitchell D, Kadlubar FF, and McManus ME (2008) Sulfotransferase 4A1. Int J Biochem Cell Biol 40:2686-2691.

Mitchell DJ, Butcher NJ, and Minchin RF (2011) Phosphorylation/dephosphorylation of human SULT4A1: role of Erk1 and PP2A. Biochim Biophys Acta 1813:231-237.

Mitchell DJ and Minchin RF (2009) Cytosolic Aryl sulfotransferase 4A1 interacts with the peptidyl prolyl cis-trans isomerase Pin1. Mol Pharmacol 76:388-395.

Nakamura J, Mizuma T, Hayashi M, and Awazu S (1990) Correlation of phenol sulphotransferase activities in the liver and platelets of rat. J Pharm Pharmacol 42:207-208.

Petrotchenko EV, Pedersen LC, Borchers CH, Tomer KB, and Negishi M (2001) The dimerization motif of cytosolic sulfotransferases. FEBS Lett 490:39-43.

Ramsey TL, Meltzer HY, Brock GN, Mehrotra B, Jayathilake K, Bobo WV, and Brennan MD (2011) Evidence for a SULT4A1 haplotype correlating with baseline psychopathology and atypical antipsychotic response. Pharmacogenomics 12:471-480.

Riches Z, Stanley EL, Bloomer JC, and Coughtrie MW (2009) Quantitative evaluation of the expression and activity of five major sulfotransferases (SULTs) in human tissues: the SULT "pie". Drug Metab Dispos 37:2255-2261.

Salman ED, Kadlubar SA, and Falany CN (2009) Expression and localization of cytosolic sulfotransferase (SULT) 1A1 and SULT1A3 in normal human brain. Drug Metab Dispos 37: 706-709.

Sidharthan NP, Butcher NJ, Mitchell DJ, and Minchin RF (2014) Expression of the orphan cytosolic sulfotransferase SULT4A1 and its major splice variant in human tissues and cells: dimerization, degradation and polyubiquitination. PLoS One 9:e101520.

Sidharthan NP, Minchin RF, and Butcher NJ (2013) Cytosolic sulfotransferase 1A3 is induced by dopamine and protects neuronal cells from dopamine toxicity: role of D1 receptor-N-methyl-Daspartate receptor coupling. J Biol Chem 288:34364-34374.

Strott CA (2002) Sulfonation and molecular action. Endocr Rev 23:703-732.

Sun M and Leyh TS (2010) The human estrogen sulfotransferase: a half-site reactive enzyme. Biochemistry 49:4779-4785.

Thomae BA, Eckloff BW, Freimuth RR, Wieben ED, and Weinshilboum RM (2002) Human sulfotransferase SULT2A1 pharmacogenetics: genotype-to-phenotype studies. Pharmacogenomics $J$ 2:48-56.

Tibbs ZE, Rohn-Glowacki KJ, Crittenden F, Guidry AL, and Falany CN (2015) Structural plasticity in the human cytosolic sulfotransferase dimer and its role in substrate selectivity and catalysis. Drug Metab Pharmacokinet 30:3-20.

Veronese ME, Burgess W, Zhu X, and McManus ME (1994) Functional characterization of two human sulphotransferase cDNAs that encode monoamine- and phenol-sulphating forms of phenol sulphotransferase: substrate kinetics, thermal-stability and inhibitor-sensitivity studies. Biochem J 302:497-502.

Address correspondence to: Dr. Neville J. Butcher, School of Biomedical Sciences, University of Queensland, St. Lucia, QLD 4072, Australia. E-mail: n. butcher@uq.edu.au 